\title{
PHYSICAL THERAPY AFFECTS ENDOTHELIAL FUNCTION IN LYMPHEDEMA PATIENTS
}

\author{
B. Brix, G. Apich, C. Ure, A. Roessler, N. Goswami
}

Physiology Division (BB,AR,NG), Otto Loewi Research Center, Gravitational Physiology and Medicine Research Unit, Medical University of Graz; Wolfsberg Clinical Center for Lymphatic Disorders (GA,CU), Wolfsberg State Hospital, KABEG, Austria

\section{ABSTRACT}

Lymphedema arises due to a malfunction of the lymphatic system and can lead to massive tissue swelling. Complete decongestive therapy (CDT), consisting of manual lymphatic drainage (MLD) and compression bandaging, is aimed at mobilizing fluid and reducing volume in affected extremities. Lymphatic dysfunction has previously been associated with chronic inflammation processes. We investigated plasma $A D M A$ as an indicator of endothelial function/inflammation before-, during- and after-CDT. Also assessed were vascular function parameters such as carotid-femoral pulse wave velocity ( $P W V c f)$, flow-mediated dilatation (FMD) and retinal microvasculature analysis. 13 patients ( 3 males and 10 females, 57 \pm 8 years old $($ mean $\pm S D), 167.2 \pm 8.3 \mathrm{~cm}$ height, $91.0 \pm 23.5 \mathrm{~kg}$ weight), with lower limb lymphedema were included. Vascular function parameters were assessed on day 1, 2, 7, 14 and 21 of CDT, pre- and post-MLD. ADMA was significantly lower post-MLD $(p=0.0064)$ and tended to reduce over three weeks of therapy $(p=0.0506)$. $P W V$ cf weakly correlated with FMD (r=0.361, $p=0.010)$. PWVcf, FMD and retinal microvasculature analysis did not show changes due to physical therapy. The novel results from this study indicate that lymphedema does not affect endothelial function and lymphedema patients may therefore not have a higher risk of cardiovascular diseases. Our results further suggest that manual lymphatic drainage with or without full CDT could have potentially beneficial effects on endothelial function in lymphedema patients (by reducing ADMA levels), which has not been reported previously.

Keywords: lymphedema, lymphedema therapy, vascular function, complex decongestive therapy, manual lymphatic drainage, ADMA (asymmetric dimethylarginine)

Lymphedema is a progressive disease with an underlying dysfunctional lymphatic vasculature. It is associated with an accumulation of excessive fluid, which can arise due to an imbalance between plasma filtration and the lymphatic outflow/transport capacity (1). No definite cure is currently available for lymphedema. Therefore, it requires continuous therapy including meticulous care on behalf of the patient, non-invasive treatment approaches and occasional surgery (1). Complete decongestive therapy (CDT) is performed to reduce volume of lymphedematous tissue/body parts, to retain the disease in a low stage and to prevent the progression of lymphedema (2). CDT is a multicomponent form of physical therapy and consists of manual lymphatic drainage (MLD), bandaging and compression of the affected body part to get control over the swelling. It also incorporates additional physical exercises such as walking, ergometry or gymnastics. CDT is known to mobilize fluid and is further believed to enhance lymphatic outflow (3). 
Deranged lymphatic flow, as seen in lymphedema patients, can result in chronic inflammatory processes (4). Recent research has linked the lymphatic system and lymphedema to inflammation, dyslipidemia, obesity, hypertension, metabolic syndrome or atherosclerosis (5-7). All these factors have been implicated in the development of cardiovascular diseases (4).

The endothelial cells lining blood vessels, by releasing vasodilators such as nitric oxide (NO) and prostacyclin (PGI2) as well as vasoconstrictors such as endothelin (ET) and platelet-activating factor (PAF), are involved in the regulation of blood flow and thus blood pressure regulation. In addition, shear stress on the inner layer of the vessel is one of the key stimulants activating endothelial nitric oxide synthase (eNOS). Activated eNOS initiates the release of NO. Due to the increase in NO, dilatation occurs in large arteries. Endothelial dysfunction, meaning the inability of vascular dilatation, is characterized by reduced levels of NO. Consequences of endothelial dysfunction can be the development of atherosclerosis, hypertension, and other cardiovascular diseases (8). As endothelial dysfunction can only be reversed in early stages, early detection is recommended. Routinely, asymmetric dimethylarginine (ADMA), an inhibitor of eNOS, is used as a marker in the plasma for the assessment of endothelial (dys-)function. Increased levels of ADMA indicate an impaired endothelial function and are used as a factor to predict cardiovascular risk (9).

As outlined above, lymphatic dysfunction is associated with chronic inflammatory processes (1,10-12), and as ADMA is an indicator of endothelial function $(13,14)$, we investigated ADMA before-, during-, and after- CDT. We hypothesized that i) endothelial dysfunction would be present at baseline in lymphedema patients, and ii) endothelial function would be improved due to the manual lymphatic drainage and/or over three weeks of decongestive therapy. To our knowledge, no previous study has investigated endothelial function changes in lymphedema patients and how it is altered during physical therapy (that is, following three weeks of
CDT). Furthermore, as ADMA is a gold standard for endothelial function assessment, its relationship with other markers of endothelial- and vascular- (dys-)function assessments such as carotid-femoral pulse wave velocity (PWVcf) and brachial flow mediated dilatation (FMD) $(15,16)$ were also investigated. We hypothesized that these markers of endothelial and vascular function will be correlated with ADMA levels. Finally, as changes in brachial and femoral arteries are expected to occur much later in disease states than in smaller arteries, retinal microvasculature changes were also assessed (16-18). We hypothesized that retinal microvascular changes would precede brachial and femoral artery changes in lymphedema patients.

\section{MATERIALS AND METHODS}

This study was performed at the Wolfsberg Clinical Center for Lymphatic Disorders, Wolfsberg State Hospital, Austria. The collected data was then analyzed at the Medical University of Graz, Austria. The study was submitted to and approved by the Ethics Committee of Carinthia (EK: A 03/17) as well as the Ethics Committee of the Medical University Graz, Austria (EK: 29-090 ex 16/17). Data collection was performed in accordance with good clinical practices and following the WMA Declaration of Helsinki (2013). Every patient received detailed information about the study protocol and provided written consent.

\section{Patients}

13 patients ( 3 males and 10 females, $57 \pm$ 8 years of age, $167.2 \pm 8.3 \mathrm{~cm}$ height, $91.0 \pm$ $23.5 \mathrm{~kg}$ weight, BMI: $32.6 \pm 6.8$, resting systolic blood pressure: $130 \pm 20 \mathrm{mmHg}$, resting diastolic blood pressure: $90 \pm 14$ $\mathrm{mmHg}$ ) with primary or secondary lymphedema took part in this study. All patients were diagnosed with stage II lower limb lymphedema [defined as pitting edema and not reversible upon elevation of the limb (19)]. Excluded were patients with any signs of mental disorders, history of cardiovascular diseases, syncope, or alcoholism. Pregnant 


\section{Data Collection during Complete Decongestive Therapy}

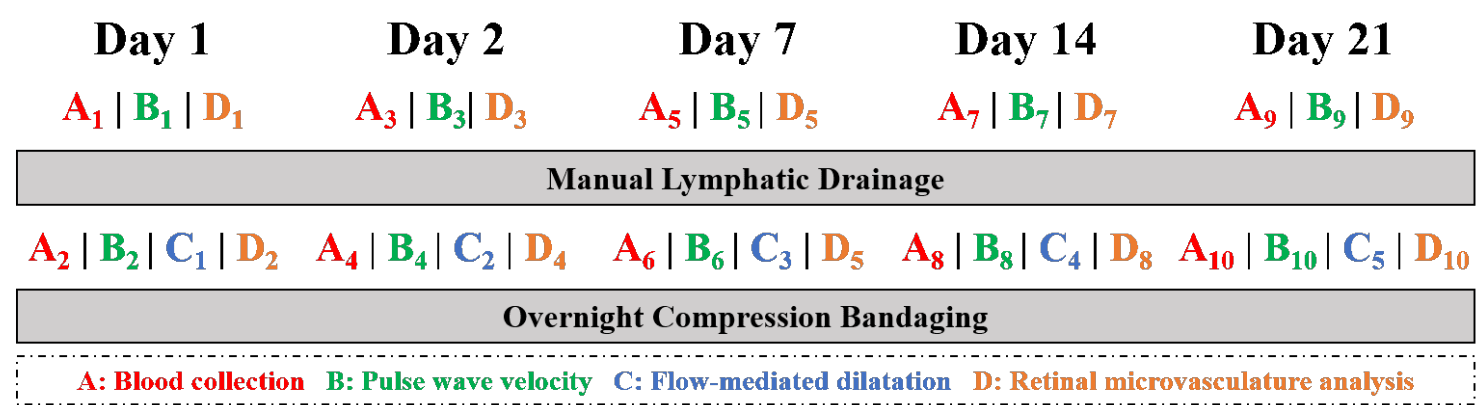

Fig. 1. Detailed study protocol. Endothelial and vascular function assessments were performed on day 1, day 2, day 7, day 14 and day 21 of complete decongestive therapy (CDT), before - and after - manual lymphatic drainage (MLD).

women were also excluded from participation.

\section{Therapy Protocol}

The physical therapy standards used at the Clinical Center for Lymphatic Disorders in Wolfsberg are following the recommendations by Döller (2). The same procedures were used in this study. Complete Decongestive Therapy lasted for three weeks. This physical therapy comprised of different phases, which were a) manual lymphatic drainage and $b$ ) the application of compression garments. During these three weeks, every weekday patients received MLD for 30 minutes. This included distinct motions conducted by specialized lymph therapists, such as flowing, rhythmic, stirring as well as scrubbing gestures at low pressure (approximately 30-40 $\mathrm{mmHg}$ ). These slow movements were adjusted to the lymphangion's spontaneous frequency of around 10 contractions per minute. Additionally, skin and subcutis were stretched during this procedure. MLD intervention was commenced in the area of healthy tissue and then expanded towards the lymphedematous tissue. Following MLD, multilayered compression bandages were applied to the affected limbs (20). Patients wore these bandages throughout the whole day and overnight. While wearing the compression bandages, physical exercises such as ergometry, gymnastics and/or walking were performed. These were aimed at improving muscle pump - as well as joint function. Finally, psychological support as well as educational seminars on skin care, nutrition and self-care were also provided as part of CDT.

\section{Study Protocol}

In order to determine the effects of our regimen of physical therapy on endothelial and vascular function parameters, measurements were performed on day $1,2,7,14$, and 21 of CDT (Fig. 1). On each of these sampling days blood collection, PWVcf, and retinal microvasculature images were performed before and after 30 minutes of MLD. FMD was assessed post-MLD on the indicated measurement days.

\section{Blood Sample Collection}

Blood was collected from patients in seated positions. EDTA blood samples were immediately stored on ice and centrifuged at $1,500 \mathrm{G}$ for 15 minutes at $4^{\circ} \mathrm{C}$. Plasma aliquots were stored at $-80^{\circ} \mathrm{C}$ until further measurements. Plasma ADMA was measured using a commercially available ELISA Kits. Due to the fact that plasma volume increases occurred as a result of the therapy, ADMA values were corrected according to the Van 
Beaumont equation (21).

\section{Vascular Function Assessments}

Carotid-femoral pulse wave velocity (PWVcf), an index for arterial stiffness, was determined using the Vicorder ${ }^{\circledR}$ device (SMT medical GmbH \& Co. KG, Germany). To assess PWVcf, one blood pressure cuff was fixed at the level of the carotid artery, whereas the second cuff was positioned as high as possible around the right thigh in supine position. In order to assess PWVcf in meters per second, the distance between the suprasternal notch and the center of the femoral cuff was also measured (22).

Flow-mediated dilatation (FMD) was performed strictly according to the guidelines described by Thijssen and colleagues (23). Patients were asked to remain supine and keep the right arm at $80^{\circ}$ in a comfortable and relaxed forearm position. Assessed were percentage changes in brachial artery diameter between baseline and maximum vessel diameter during hyperemia. This was induced by inflation and deflation of asphygmomanometer cuff to suprasystolic levels for 5 minutes.

To assess retinal microvascular changes, images of both eyes were obtained using a hand-held smartscope Pro Fundus camera (Optomed, Finland). Optic disc-centered images were analyzed via the iFlexis software (VITO, Belgium). Diameter averages of the six largest arterioles and venules between 0.5 - and 1-disc diameters from the optic disc margin were analyzed (17). Data are presented as central retinal arteriolar equivalent (CRAE) and central retinal venular equivalent (CRVE). Finally, arteriolar-to-venous ratio (A-to-V ratio) was calculated (for details see (16-18,24).

\section{Statistics}

Data are presented as mean \pm standard deviation (SD). Analyses of variances (twoway-ANOVA) were performed to determine effects over three weeks of therapy and due to lymphatic drainage (MLD). Correlations were explored by Pearson's correlation. P-values $<0.05$ were considered as statistically significant. All data were analyzed using SPSS (Vers. 26.0, SPSS Inc., USA). GraphPad Prism (Vers. 8, GraphPad Software, Inc., USA) was used to generate the figures.

\section{RESULTS}

13 patients ( 3 males and 10 females, $57 \pm 8$ years of age, $167.2 \pm 8.3 \mathrm{~cm}$ height, $91.0 \pm 23.5$ kg weight) with primary or secondary lymphedema were included in the study.

Plasma ADMA decreased significantly due to MLD from $0.54 \pm 0.02 \mu \mathrm{mol} / \mathrm{l} \mathrm{pre}-\mathrm{MLD}$ to $0.49 \pm 0.02 \mu \mathrm{mol} / 1$ post- $\mathrm{MLD}\left(\mathrm{F}_{(1 ; 6)}=16.75\right.$, $\mathrm{p}=0.0064)$. Plasma ADMA levels tended to reduce over three weeks of CDT $\left(\mathrm{F}_{(4 ; 24)}=2.76\right.$, $\mathrm{p}=0.0506$ ) from $0.54 \pm 0.02 \mu \mathrm{mol} / \mathrm{l}$ to $0.49 \pm$ $0.02 \mu \mathrm{mol} / \mathrm{l}$ (Table 1; Fig. 2).

Pearson's correlation showed a significant but weak correlation between PWVcf and FMD ( $r=0.361, p=0.01)$. However, no changes were seen in $P W V c f\left(F_{(1 ; 7)}=0.05 ; p=0.8302\right)$ and retinal image analysis due to MLD (CRAE: $\mathrm{F}_{(1 ; 5)}=2.20 ; \mathrm{p}=0.1981$; CRVE: $F_{(1 ; 5)}=0.25 ; p=0.6401$ and A-to-V: $F_{(1 ; 5)}=1.15$; $\mathrm{p}=0.3318$ ) (Table 1). In addition, PWVcf $\left(\mathrm{F}_{(4 ; 28)}=0.34 ; \mathrm{p}=0.8478\right)$, FMD $\left(\mathrm{F}_{(4 ; 32)}=1.41\right.$; $\mathrm{p}=0.252)$ as well as retinal microvasculature analysis $\left(\mathrm{CRAE}: \mathrm{F}_{(4 ; 2)}=1.47 ; \mathrm{p}=0.2488\right.$; CRVE: $\mathrm{F}_{(4 ; 20)}=2.55 ; \mathrm{p}=0.0708$ and A-to-V: $\left.F_{(4 ; 20)}=2.16 ; p=0.1104\right)$ did not show any changes over three weeks of therapy (Table 1).

\section{DISCUSSION}

We observed that the gold standard of endothelial function assessment (ADMA) was not elevated in lymphedema patients. Further, manual lymphatic drainage led to significantly reduced plasma ADMA levels $(\mathrm{p}=0.0064)$. A significant correlation $(\mathrm{p}=0.01)$ was found between pulse wave velocity and flow-mediated dilatation measurements. However, no changes in pulse wave velocity, flow mediated dilatation, and retinal microvasculature (as indicator of microvascular health) were observed due to physical therapy in lymphedema patients. Plasma ADMA may be a 


\section{TABLE 1}

An Overview of Endothelial and Vascular Function Parameters (mean \pm SD) before - and after - Manual Lymphatic Drainage, Over Three Weeks of Physical Therapy.

Blood samples for ADMA analysis were obtained from 7 patients, PWVef was determined in 8 patients, FMID in 9 patients and retinal microvasculature analysis was performed in 6 patients

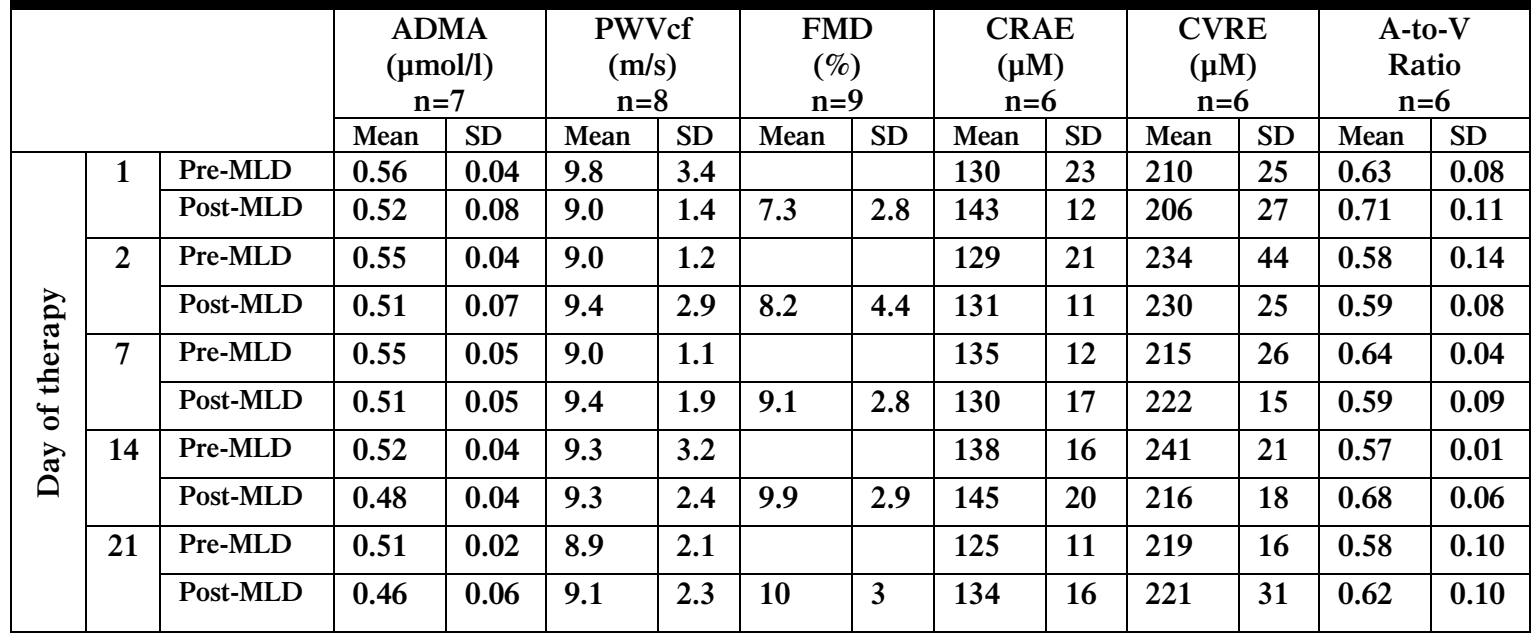

ADMA, asymmetric dimethylarginine; A-to-V, arteriolar-to-venous ratio; CDT, complete decongestive therapy; $C R A E$, central arteriolar equivalent; CRVE, central retinal venular equivalent; MLD, manual lymphatic drainage; $P W V c f$, carotid-femoral pulse wave velocity.

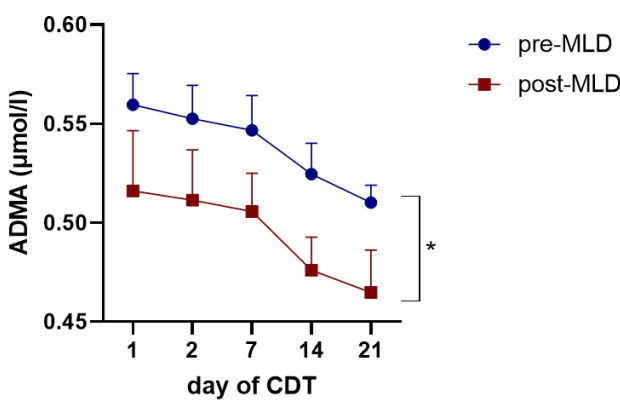

Fig. 2. Time-course of plasma asymmetric dimethylarginine (ADMA) levels ( $\mu \mathrm{mol} / \mathrm{l})$ due to manual lymphatic drainage $(M L D)$ and over three weeks of complete decongestive therapy $(C D T)$. The blue circles represent ADMA levels pre-MLD, whereas the red boxes show ADMA levels post$M L D$, over three weeks of physical therapy. The asterisk (*) represents significant difference between pre-MLD and post-MLD ADMA measurements ( $p=0.0064)$. Legend: ADMA, asymmetric dimethylarginine; $C D T$, complete decongestive therapy, MLD, manual lymphatic drainage. sensitive indicator for minor changes in endothelial health in lymphedema patients. This is not surprising as ADMA is the gold standard for endothelial function $(13,25,26)$.

We hypothesized that lymphedema patients already show signs of endothelial dysfunction at baseline. However, we found that plasma ADMA levels were within the normal range at baseline. Baseline ADMA in our study was $0.56 \pm 0.04 \mu \mathrm{mol} / \mathrm{l}$, which is within the range of plasma ADMA seen in healthy persons $(0.25-0.92 \mu \mathrm{mol} / \mathrm{l})(9)$. Thus, it appears that patients with lymphedema do not have endothelial dysfunction. To our knowledge, these are novel results, as we could not find any study that documented specifically endothelial function in lymphedema patients. As endothelial dysfunction is associated with higher risk of cardiovascular diseases (14), our results suggest that lymphedema patients generally may not have a higher cardiovascular risk. 
Another finding was related to the effects of this form of physical therapy. We observed that $\mathbf{3 0}$ minutes of manual lymphatic drainage leads to a significant decrease in ADMA from $0.54 \pm 0.02 \mu \mathrm{mol} / 1$ to $0.49 \pm 0.02 \mu \mathrm{mol} / \mathrm{l}$ $(\mathrm{p}=0.0064)$. There are limited studies examining the short-term effects of different interventions/ perturbations on plasma ADMA. Zinellu et al. (2017), for example, investigated plasma ADMA after a single session of exercise and found that ADMA significantly decreased post-exercise by $8 \%$ (27). Although Zinellu and colleagues used a much more strenuous intervention, we found similar effects ( $8 \%$ vs 9\% decrease) in our study, in which lymphatic drainage was performed. This indicates that manual lymphatic drainage has a beneficial effect on endothelial function in lymphedema patients. To our knowledge we are not aware of any study that has specifically investigated how manual lymphatic drainage effects endothelial health. Although the baseline values were not elevated, our results indicate that manual lymphatic drainage could have potentially beneficial affects on endothelial function in lymphedema patients. To our knowledge, we are not aware of any study that has specifically investigated how manual lymphatic drainage effects markers of endothelial (dys)function. However, this issue needs to be further evaluated in future studies including more lymphedema patients with different stages of lymphedema as well as different duration of lymphedema, as this could also affect endothelial (dys-)function and predispose these patients to higher risk of cardiovascular diseases.

Additionally, we also assessed whether decongestive therapy over three weeks affects endothelial health. Our results show that ADMA tended to reduce over three weeks of physical therapy $(p=0.0506)$. Since we are not aware of any study that has specifically investigated ADMA over three weeks of decongestive complete therapy, we examined other studies in which ADMA was measured over varying interventions. For example, Rudofsky et al observed a reduction of ADMA levels during a weight loss therapy program of 12 weeks from $0.47 \pm 0.07$ to $0.42 \pm 0.08 \mathrm{mmol} / \mathrm{l}$
(28). Another study reported reductions in ADMA concentrations (from $0.54 \pm 0.02$ to $0.44 \pm 0.03 \mu \mathrm{mol} / \mathrm{l})$ after two months of physical exercise training in type 1 diabetes patients (29).

Pulse wave velocity and flow mediated dilatation did not change due to manual lymphatic drainage nor over three weeks of physical therapy (Table 1). As these methods assess vascular changes in rather large arteries (carotid artery, femoral artery, and brachial artery) (16), it is possible that assessment of these arteries is not sensitive enough to detect endothelial function changes in lymphedema patients. Or, it could be that such changes in endothelial/vascular function might only appear in later stages of the disease.

We observed that ADMA did not have any relationship with pulse wave velocity ( $r=$ $-0.044, p=0.739)$ and flow-mediated dilatation $(r=-0.112, p=0.550)$. This is surprising, as previous reports have shown an association between these measurements of endothelial health and vascular function assessment $(26,30,31)$. Protopsaltis and colleagues observed a correlation between ADMA and pulse wave velocity in prediabetic patients (31). An inverse correlation between ADMA and flow-mediated dilatation was previously reported in healthy individuals (32), prediabetic women (33) and patients with rheumatoid arthritis (30). While we could not confirm such associations in these measurements in our study, our results show that there is a correlation between pulse wave velocity and flow-mediated dilatation ( $\mathrm{p}=\mathbf{0 . 0 1 0})$. This is in agreement with previous reports that have shown such correlations $(16,34,35)$.

Despite retinal measurement being suggested as indicator of microvascular health (16-18), we could not find any changes in the ophthalmic vessels in lymphedema patients nor following lymphatic drainage nor as a result of three weeks of physical therapy. In addition, retinal microvascular parameters did not show any correlation to ADMA. Although a previous study reported spastic and stagnant microcirculation in lymphedema patients (measured by laser Doppler flowmetry in the legs), as well as improvement of such follow- 
ing physical therapy (36), we could not observe such microvascular changes in the retina in our study. It is possible that the microvascular changes in the retina occur much later (or are absent) in our patients, as most of them had localized lymphedema in the legs. Future research should investigate whether vascular function changes occur in different vascular beds or differ depending on the involved regions in lymphedema patients.

A possible limitation of this study is the small number of patients that were included $(n=13)$. However, even with the low number of patients participating in this study, we could show significant changes in endothelial function by assessing plasma ADMA. In addition, we believe that the strength of our study is that each person was his/her own control and the same measurements were carried out over time in the same persons. As this was an exploratory study, we will now use the data obtained here to carry out sample size calcula-tions for larger epidemiological studies that will investigate how endothelial function is affected with and without physical therapy in lymphedema patients. Another limitation of this study is that there was a far larger number of females enrolled in our study as compared to males. We do not think this is a limitation as females are most affected by this chronic debilitating disorder (37).

\section{CONCLUSIONS AND FUTURE DIRECTIONS}

Our novel results indicate a beneficial effect of manual lymphatic drainage as it led to reductions in plasma AMDA levels (reflecting improvements in endothelial health) in lymphedema patients. As other markers of endothelial (dys-)function and vascular health (e.g. pulse wave velocity, flow-mediated dilatation, or retinal microvascula-ture analysis) did not show changes due to physical therapy, plasma ADMA may be the preferred biomarker to assess endothelial function changes in lymphedema patients. Future studies should examine how these markers of endothelial - and vascular - health are modulated by types of lymphedema (primary vs. secondary), in different body parts (arm vs. leg vs. more generalized) or by varying therapies.

\section{List of Abbreviations}

ADMA: Asymmetric dimethylarginine

A-to-V: Arteriolar-to-venous ratio

CDT: Complete decongestive therapy CRAE: Central retinal arteriolar equivalent CRVE:

Central retinal venular equivalent eNOS:

Endothelial nitric oxide synthase

ET: Endothelin

FMD: Flow-mediated dilatation

MLD: Manual lymphatic drainage

PWVcf: Carotid-femoral pulse wave velocity

NO: Nitric oxide

PAF: Platelet-activating factor

PGI2: Prostacyclin

\section{ACKNOWLEDGMENTS}

We wish to thank all patients for their time and willingness to take part in this study. All authors have made significant contributions in study planning, data collection, analysis as well as interpretation. All co-authors have been involved in writing the final version of the manuscript.

\section{CONFLICT OF INTEREST AND DISCLOSURE}

The authors declare no competing financial interests exist.

\section{REFERENCES}

1. Hu, D, L Li, S Li, et al: Lymphatic system identification, pathophysiology and therapy in the cardiovascular diseases. J. Mol. Cell Cardiol. 133 (2019), 99-111.

2. Döller, W: Lymphologie. Wiener Medizinische Wochenschrift. 163 (2013), 153-154.

3. Földi, E, A Sauerwald, B Hennig: Effect of complex decongestive physiotherapy on gene expression for the inflammatory response in peripheral lymphedema. Lymphology 33 (2000), 151-157.

4. Telinius, N, VE Hjortdal: Role of the 
lymphatic vasculature in cardiovascular medicine. Heart 105 (2019), 1777-1784.

5. Ho, YC, RS Srinivasan: Lymphatic vasculature in energy homeostasis and obesity. Front. Physiol. 11 (2020), 3.

6. Dayan, JH, CL Ly, RP Kataru, et al: Lymphedema: Pathogenesis and novel therapies. Ann. Rev. Med. 69 (2018), 263-276.

7. Kataru, RP, JE Baik, HJ Park, et al: Regulation of immune function by the lymphatic system in lymphedema. Front. Immunol. 10 (2019), 470.

8. Konukoglu, D, H Uzun: Endothelial dysfunction and hypertension. Adv. Exp. Med. Biol. 956 (2017), 511-540.

9. Nemeth, B, Z Ajtay, L Hejjel, et al: The issue of plasma asymmetric dimethylarginine reference range - A systematic review and meta-analysis. PLoS One 12 (2017), e0177493.

10. Aspelund, A, MR Robciuc, S Karaman, et al: Lymphatic system in cardiovascular medicine. Circ. Res. 118 (2016), 515-530.

11. Chakraborty, S, S Zawieja, W Wang, et al: Lymphatic system: A vital link between metabolic syndrome and inflammation. Ann. New York Acad. Sci. 1207 (2010), E94-E102.

12. Csanyi, G, B Singla: Arterial lymphatics in atherosclerosis: Old questions, new insights, and remaining challenges. J. Clin. Med. 8 (2019), 495.

13. Cooke, JP: ADMA: Its role in vascular disease. Vasc. Med. 10 (2005), S11-17.

14. Sibal, L, SC Agarwal, PD Home, et al: The role of asymmetric dimethylarginine (ADMA) in endothelial dysfunction and cardiovascular disease. Curr. Cardiol. Rev. 6 (2010), 82-90.

15. Strijdom, H, S Charania, N Goswami, et al: Cardiovascular health and flow-mediated dilatation (FMD) In a south african cohort of $\mathrm{HIV}$-infected participants-findings from the endoafrica study. Atherosclerosis 263 (2017), E141.

16. Strijdom, H, P De Boever, G Walzl, et al: Cardiovascular risk and endothelial function in people living with HIV/AIDS: Design of the multi-site, longitudinal EndoAfrica study in the Western Cape Province of South Africa. BMC Infect. Dis. 17 (2017), 41.

17. Louwies, T, L Int Panis, T Alders, et al: Microvascular reactivity in rehabilitating cardiac patients based on measurements of retinal blood vessel diameters. Microvasc. Res. 124 (2019), 25-29.

18. Vaes, AW, MA Spruit, J Theunis, et al: Looking into the eye of patients with chronic obstructive pulmonary disease: An opportunity for better microvascular profiling of these complex patients. Acta Ophthalmol. 96 (2018), 539-549.

19. Kayıran, O, C De La Cruz, K Tane, et al: Lymphedema: From diagnosis to treatment. Turk. J. Surg. 33 (2017), 51-57.

20. Apich, G: Konservative therapie des lymphoedems - Lymphologische rehabilitationsbehandlung. Wiener Medizinische Wochenschrift. 163 (2013), 169-176.

21. Van Beaumont, W: Evaluation of hemoconcentration from hematocrit measurements. J. Appl. Physiol. 32 (1972), 712-713.

22. Koivistoinen, T, M Virtanen, N HutriKahonen, et al: Arterial pulse wave velocity in relation to carotid intima-media thickness, brachial flow-mediated dilation and carotid artery distensibility: The cardiovascular risk in young Finns study and the health 2000 survey. Atherosclerosis 220 (2012), 387-393.

23. Thijssen, DHJ, RM Bruno, A van Mil, et al: Expert consensus and evidence-based recommendations for the assessment of flowmediated dilation in humans. Eur. Heart $\mathbf{J}$. 40 (2019), 2534-2547.

24. Matušková, V, T Zeman, L Ewerlingová, et al: An association of neovascular age-related macular degeneration with polymorphisms of CFH, ARMS2, HTRA1 and C3 genes in Czech population. Acta Ophthalmol. 98 (2020), E691-699.

25. Deneva-Koycheva, TI, LG VladimirovaKitova, EA Angelova, et al: Plasma asymmetric dimethylarginine levels in healthy people. Folia Med. (Plovdiv). 53 (2011), 28-33.

26. Baum, C, SS Johannsen, T Zeller, et al: ADMA and arginine derivatives in relation to non-invasive vascular function in the general population. Atherosclerosis 244 (2016), 149-156.

27. Zinellu, A, S Sotgia, MF Usai, et al: Plasma ADMA decrease following a single bout of physical exercise is related to reduced homocysteine lowering. Pharmaceutical Sciences and Biomedical Analysis Journal. 1 (2017), 112.

28. Rudofsky, G, E Roeder, T Merle, et al: Weight loss improves endothelial function independently of ADMA reduction in severe obesity. Horm Metab. Res. 43 (2011), 343-348.

29. Mittermayer, F, J Pleiner, K Krzyzanowska, et al: Regular physical exercise normalizes elevated asymmetrical dimethylarginine concentrations in patients with type 1 
diabetes mellitus. Wien Klin. Wochenschr. 117 (2005), 816-820.

30. Şentürk, T, N Yılmaz, G Sargın, et al: Relationship between asymmetric dimethylarginine and endothelial dysfunction in patients with rheumatoid arthritis. Eur. J. Rheumatol. 3 (2016), 106-108.

31. Protopsaltis, I, S Foussas, A Angelidi, et al: Impact of ADMA, endothelial progenitor cells and traditional cardiovascular risk factors on pulse wave velocity among prediabetic individuals. Cardiovasc. Diabetol. 11 (2012), 141.

32. Ardigo, D, M Stüehlinger, L Franzini, et al: ADMA is independently related to flowmediated vasodilation in subjects at low cardiovascular risk. Eur. J. Clin. Invest. 37 (2007), 263-269.

33. Eliana, F, P Suwondo, LH Makmun, et al: AADMA as a marker of endothelial dysfunction in prediabetic women. Acta Med. Indones. 43 (2011), 92-98.

34. Soltész, P H Dér, G Kerekes, et al: A comparative study of arterial stiffness, flowmediated vasodilation of the brachial artery, and the thickness of the carotid artery intima-media in patients with systemic autoimmune diseases. Clin. Rheumatol. 28 (2009), 655-662.
35. Figueiredo, VN, JC Yugar-Toledo, LC Martins, et al: Vascular stiffness and endothelial dysfunction: Correlations at different levels of blood pressure. Blood Press. 21 (2012), 31-38.

36. Apkhanova, TV, DB Kulchitskaya, SV Sapelkin: [A microcirculatory change induced by comprehensive anti-edematous therapy in patients with lower limb lymphedema]. Vopr. Kurortol. Fizioter. Lech Fiz. Kult. 96 (2019), 9-15.

37. Keeley, V, P Franks, I Quere, et al: LIMPRINT in specialist lymphedema services in United Kingdom, France, Italy, and Turkey. Lymph. Res. Bio. 17 (2019), 141-146.

Dr. Nandu Goswami, MBBS, PhD, Master of Medical Science (Major in Medical Education) Assoc. Prof. , Head of Research Unit "Gravitational Physiology and Medicine" Acting Chair of Physiology, Otto Loewi Research Center, Medical University of Graz Neue Stiftingtalstraße 6/D.05 A-8010 Graz, Austria

Tel: +43316 38573852

Fax: +4331638579005

E-mail: nandu.goswami@medunigraz.at 